University of Nebraska - Lincoln

DigitalCommons@University of Nebraska - Lincoln

May 2007

\title{
LABORATORY FINDINGS IN ACUTE CYTAUXZOON FELIS INFECTION IN COUGARS (PUMA CONCOLOR COUGUAR) IN FLORIDA
}

\author{
John W. Harvey \\ University of Florida \\ Mike R. Dunbar \\ Florida Game and Fresh Water Fish Commission \\ Terry M. Norton \\ University of Florida \\ Michael J. Yabsley \\ University of Georgia, Athens
}

Follow this and additional works at: https://digitalcommons.unl.edu/icwdm_usdanwrc

Part of the Environmental Sciences Commons

\begin{abstract}
Harvey, John W.; Dunbar, Mike R.; Norton, Terry M.; and Yabsley, Michael J., "LABORATORY FINDINGS IN ACUTE CYTAUXZOON FELIS INFECTION IN COUGARS (PUMA CONCOLOR COUGUAR) IN FLORIDA" (2007). USDA National Wildlife Research Center - Staff Publications. 690.

https://digitalcommons.unl.edu/icwdm_usdanwrc/690
\end{abstract}

This Article is brought to you for free and open access by the U.S. Department of Agriculture: Animal and Plant Health Inspection Service at DigitalCommons@University of Nebraska - Lincoln. It has been accepted for inclusion in USDA National Wildlife Research Center - Staff Publications by an authorized administrator of DigitalCommons@University of Nebraska - Lincoln. 


\title{
LABORATORY FINDINGS IN ACUTE CYTAUXZOON FELIS INFECTION IN COUGARS (PUMA CONCOLOR COUGUAR) IN FLORIDA
}

\author{
John W. Harvey, D.V.M., Ph.D., Dipl. A.C.V.P., Mike R. Dunbar, M.S., D.V.M., Terry M. \\ Norton, D.V.M, Dipl. A.C.Z.M., and Michael J. Yabsley, M.S., Ph.D.
}

\begin{abstract}
Intraerythrocytic piroplasms, morphologically indistinguishable from Cytauxzoon felis, were identified in stained blood films from more than one third of free-ranging cougars (Puma concolor couguar) in southern Florida in a study that failed to demonstrate negative effects of piroplasm infection on measured hematologic parameters. However, a recent study with a nested 18s rRNA polymerase chain reaction (PCR) assay identified only $9 \%$ of the free-ranging cougars in southern Florida as infected with $C$. felis but found $83 \%$ of these animals were infected with an unnamed small Babesia sp. In this study, hematology and clinical chemistry parameters were determined during the initial appearance of piroplasms in stained blood films of three western cougars housed in northern Florida. One animal became ill, but the remaining two animals did not exhibit clinical signs of disease. The hematocrit decreased in all three cougars concomitant with the first recognized parasitemia. A regenerative response to anemia (increased polychromasia, increased mean cell volume, and increased red cell distribution width) was recognized in two cougars that were examined twice during the following 2 weeks. Thrombocytopenia and probable leukopenia occurred in one animal. The most consistent clinical chemistry findings were increased serum bilirubin concentrations and increased alanine aminotransferase and aspartate aminotransferase activities at the time of initial recognition of parasitemia. Serum protein findings were not consistent in these cougars. The use of PCR and determination of 18S rRNA gene sequences in the blood from these three animals revealed infection with $C$. felis, but not with the Babesia sp. In this report, we demonstrate that mild hemolytic anemia, and probably liver injury, occurs concomitant with the initial discovery of $C$. felis piroplasms in stained blood films.
\end{abstract}

Key words: Cougar, clinical biochemistry, Cytauxzoon, hematology, piroplasm, puma, Babesia.

\section{INTRODUCTION}

Cytauxzoon felis is a protozoal parasite in the family Theileriidae. Cytauxzoon differs from the genus Theileria in that schizogony occurs in macrophages rather than lymphocytes, but intraerythrocytic organisms (piroplasms) of these genera are morphologically indistinguishable. Cytauxzoonosis was first recognized as a fatal infection of domestic

From the Department of Physiological Sciences, Box 100144 (Harvey) and the Department of Small Animal Clinical Sciences, Box 100126 (Norton), College of Veterinary Medicine, University of Florida, Gainesville, Florida 32610, USA; the Florida Game and Fresh Water Fish Commission, Wildlife Research Laboratory, 4005 South Main Street, Gainesville, Florida 32601, USA (Dunbar); and the D. B. Warnell School of Forestry and Natural Resources and Southeastern Cooperative Wildlife Disease Study, Department of Population Health, College of Veterinary Medicine, University of Georgia, Athens, Georgia 30602, USA (Yabsley). Present addresses (Dunbar): U.S. Department of Agriculture, Animal and Plant Health Inspection Service, National Wildlife Research Center, 4101 LaPorte Avenue, Fort Collins, Colorado 80521, USA; (Norton): St. Catherine's Island Center, 182 Camellia Road, Midway, Georgia 31320, USA. Correspondence should be directed to Dr. Harvey (harveyj@ mail.vetmed.ufl.edu). cats (Felis catus) in southwestern Missouri, ${ }^{20}$ but this disease has now been documented in many south central and southeastern states. ${ }^{10}$

Nearly all infected domestic cats die of the disease. However, a less virulent strain of $C$. felis has been reported in a restricted area of northwestern Arkansas and northeastern Oklahoma. ${ }^{16}$ In this area, domestic cats have survived without specific treatment and four asymptomatic cats were discovered by collecting blood from healthy cats in households with ill cats.

Cytauxzoonosis is a tick-transmitted disease that occurs most frequently in rural, wooded areas inhabited by both North American bobcats (Lynx rufus) and ticks. Because domestic cats generally die of acute illness in a matter of days after becoming ill, they appear to be dead-end hosts. Bobcats, however, usually do not die when infected with $C$. felis. ${ }^{2,8}$ They have been shown to be a reservoir of infection by experimental transmission of $C$. felis to domestic cats with Dermacenter variabilis ticks infected on parasitemic bobcats. ${ }^{1}$

The only documented remaining cougar population in the eastern United States consists of fewer than 100 individuals in southern Florida. These animals have previously been called Florida panthers and classified in the subspecies Puma concolor cor- 
$y i$, but recent genetic analysis indicates that all cougars in North American belong to the same subspecies (Puma concolor couguar). ${ }^{5}$ The population is endangered because of various factors, including loss of habitat, small population size, and inbreeding resulting from isolation from other cougar populations. To improve genetic diversity, a plan was developed to release female western cougars from Texas into the areas occupied by Florida cougars. ${ }^{15}$ As part of this plan, animals were transported from Texas to Florida and quarantined in outdoor pens at the Florida Game and Freshwater Fish Commission Wildlife Research Laboratory in Gainesville, Florida.

Northern Florida is an endemic area for $C$. felis infections. Many domestic cats (Harvey, unpubl. data) and a white tiger (Panthera tigris) have become infected and died from cytauxzoonosis in northern Florida, ${ }^{7}$ as confirmed by the presence of both organisms in erythrocytes and schizonts in tissue macrophages. More than one third of the freeranging cougars in southern Florida have protozoal parasites visible in their erythrocytes in stained blood films that are identical in size and morphology to those seen in domestic cats with cytauxzoonosis. ${ }^{18}$ The presumption that these organisms were C. felis was supported by the finding that blood from an infected Florida cougar (housed in northern Florida) injected into a specific pathogen free (SPF) domestic cat, resulted in the death of the domestic cat with classical histopathologic findings of cytauxzoonosis. ${ }^{4}$ Production of fatal cytauxzoonosis by injection of blood alone is unusual. Generally only a nonfatal parasitemia results from inoculations with infected blood. ${ }^{12,13}$ Production of fatal infections usually requires feeding on cats by infected ticks or experimental inoculations with tissues containing schizonts. ${ }^{1,21}$ Schizonts develop in macrophages lining the lumen of veins; consequently, blood transmission can result in fatal disease if the injected blood contains schizonts. Fatalities attributable to $C$. felis infections have not been identified in Florida cougars. ${ }^{3,19}$

A previous study of free-ranging cougars in southern Florida failed to identify significant hematologic abnormalities attributable to chronic $C$. felis infections; ${ }^{18}$ however, a recent study with a nested 18s rRNA polymerase chain reaction (PCR) assay revealed that only $9 \%$ of the free-ranging cougars in southern Florida were infected with $C$. felis but found $83 \%$ of these animals were infected with an unnamed small Babesia. ${ }^{23}$ In this paper, we describe hematologic findings in three cougars during what is believed to be an acute infection with C. felis in northern Florida.

\section{CASE REPORTS}

A 2-yr old male western cougar (cougar 1) that was housed at a private breeding facility in northern Florida was examined at the University of Florida College of Veterinary Medicine on 21 April 1989 because of a 2-day history of anorexia and lethargy. The animal had been housed at the facility for more than $1 \mathrm{yr}$ but had been moved into a large heavily wooded pen within the previous 3 wks. The cougar was immobilized with tiletamine-zolazepam and anesthetized with isofluorane so that a physical examination, venipuncture, and radiography could be done. Physical examination revealed dehydration (estimated at 5-7\%), fair hair coat condition, and a rectal temperature of $38.9^{\circ} \mathrm{C}$. The body weight was $50 \mathrm{~kg}$, which was $5 \mathrm{~kg}$ less than that measured 11 wk earlier during a routine health assessment. Numerous Amblyomma americanum (lone star) ticks and unidentified soft ticks were found over the entire body. Ethylenediaminetetraacetic acid (EDTA)-whole blood was submitted for a complete blood count (CBC; Coulter Counter S+4, Coulter Electronic Inc., Hialeah, Florida 33010, USA) and serum for a clinical chemistry panel (550 Express Spectrophotometer and 664 Fast 4 System, CibaCorning Diagnostics, Medfield, Massachusetts 02052, USA). In addition to values measured when ill, CBC results from a previous health screen (6 February 1989) and a subsequent health screen (4 November 1989) are included in Table 1 for comparison. The hematocrit, total leukocyte count, neutrophil count, lymphocyte count, and platelet count were lower when the animal was ill compared with values before or after the illness. Serum clinical chemistry findings are given in Table 2 when the cougar was ill and from a subsequent health screen (4 November 1989). Analytes outside the reference intervals included increased serum bilirubin, alanine aminotransferase (ALT), aspartate aminotransferase (AST), and decreased serum albumin when the animal was ill. Serologic tests for feline leukemia virus and feline immunodeficiency virus were negative. Radiography of the thorax and abdomen revealed splenomegaly that was believed to be secondary to the anesthesia. Ticks were removed and the animal was treated with amoxicillin for 10 days. The subsequent finding that blood from a Florida cougar housed at this same facility transmitted fatal cytauxzoonosis to a domestic cat ${ }^{4}$ prompted a careful re-examination of archived, stained blood films from cougars. When stained blood films were examined by a veterinary clinical pathologist (JWH), small protozoal organisms consistent with $C$. felis were found in erythrocytes of 
Table 1. Hematologic findings in an adult captive cougar (cougar 1). ${ }^{\mathrm{a}}$

\begin{tabular}{lcccc}
\hline \multicolumn{1}{c}{ Parameter } & Feb 6 & Apr 21 & Nov 4 & Reference $^{\text {b }}$ \\
\hline Hematocrit $(\%)$ & 50 & 38 & 43 & $31-43$ \\
MCV $(\mathrm{fl})$ & 47 & 47 & 44 & $45-51$ \\
MCHC $(\mathrm{g} / \mathrm{dl})$ & 34 & 35 & 34 & $34 \pm 3$ \\
Platelets $\left(\times 10^{3} / \mu \mathrm{l}\right)$ & 385 & 9 & aggregates $^{\mathrm{c}}$ & $244-543$ \\
Leukocytes $\left(\times 10^{3} / \mu \mathrm{l}\right)$ & 7.6 & 4.4 & 6.7 & $8.1-15.7$ \\
Neutrophils & 4.3 & 2.5 & 3.7 & $4.0-12.5$ \\
Lymphocytes & 2.6 & 1.1 & 1.9 & $1.4-5.6$ \\
Monocytes & 0.3 & 0.5 & 0.3 & $0.1-0.8$ \\
Eosinophils & 0.4 & 0.1 & 0.8 & $0.1-0.8$ \\
Basophils & 0 & 0.1 & 0 & $0-0.2$ \\
Piroplasms in blood & neg & pos & pos & NR \\
\hline
\end{tabular}

\footnotetext{
${ }^{a} \mathrm{MCV}$, mean cell volume; MCHC, mean cell hemoglobin concentration; NR, not reported.

${ }^{b}$ Values from free-ranging adult and juvenile Florida panthers represent 10th to 90th percentile compiled with several different chemistry analyzers. ${ }^{6}$ It is unlikely that all of these reference animals were healthy.

c A platelet count was not possible because of the presence of platelet aggregates containing degranulated platelets, but it was estimated to be normal.
}

this current animal when ill (Fig. 1) and during health screens 6 mo and 1 yr later, but not during three previous health screens. Other cougars at this private breeding facility were also demonstrated to have piroplasms in erythrocytes, and a captive white tiger living in this facility died of cytaux-

Table 2. Serum clinical biochemistry findings in an adult captive cougar (cougar 1$){ }^{\mathrm{a}}$

\begin{tabular}{lccc}
\hline \multicolumn{1}{c}{ Parameter } & Apr $21^{\mathrm{b}}$ & Nov 4 & Reference $^{\mathrm{c}}$ \\
\hline Glucose $(\mathrm{mg} / \mathrm{dl})$ & 182 & 117 & $96-236$ \\
Sodium $(\mathrm{mmol} / \mathrm{L})$ & 149 & 157 & $149-157$ \\
Potassium $(\mathrm{mmol} / \mathrm{L})$ & 3.9 & 4.5 & $4.2-5.2$ \\
Chloride $(\mathrm{mmol} / \mathrm{L})$ & 114 & 125 & $110-121$ \\
Total CO $(\mathrm{mmol} / \mathrm{L})$ & 8 & 11 & $10-19$ \\
Calcium $(\mathrm{mg} / \mathrm{dl})$ & 9.0 & 10 & $9.1-10.8$ \\
Phosphate $(\mathrm{mg} / \mathrm{dl})$ & 2.9 & 4.7 & $3.7-7.8$ \\
Urea nitrogen (mg/dl) & 22 & 27 & $23-58$ \\
Creatinine (mg/dl) & 2.4 & 2.9 & $1.1-2.5$ \\
Bilirubin $(\mathrm{mg} / \mathrm{dl})$ & 1.1 & 0.2 & $0.1-0.4$ \\
Cholesterol (mg/dl) & 140 & 171 & $112-182$ \\
Total protein (g/dl) & 6.7 & 7.4 & $6.4-8.2$ \\
Albumin (g/dl) & 2.0 & 3.5 & $3.3-4.1$ \\
Globulin (g/dl) & 4.7 & 3.9 & NR \\
Alk Phos (U/L) & ND & 33 & $6-98$ \\
ALT (U/L) & 192 & 171 & $33-92$ \\
AST (U/L) & 265 & 25 & $37-106$ \\
\hline
\end{tabular}

a Alk Phos, alkaline phosphatase; ALT, alanine aminotransferase; AST, aspartate aminotransferase; ND, not detectable; NR, not reported.

${ }^{\mathrm{b}}$ Slight hemolysis was present in plasma.

c Values from free-ranging adult and juvenile Florida panthers represent 10 th to 90 th percentile compiled with several different chemistry analyzers. ${ }^{6}$ It is unlikely that all of these reference animals were healthy. zoonosis. ${ }^{7}$ Cougar 1 was subsequently housed at an environmental education center near Savannah, Georgia, until November 2005, when he was euthanized because of illness attributed to diabetes mellitus. Blood collected at the time of euthanasia was strongly positive for C. felis (and negative for the Babesia sp.) on the basis of PCR and sequencing of a fragment of the $18 \mathrm{~S}$ rRNA gene. ${ }^{23}$

Two western cougars (cougars 2 and 3) were translocated from Texas to northern Florida in March 1995 and held in quarantine before release in southern Florida. As part of the quarantine pro-

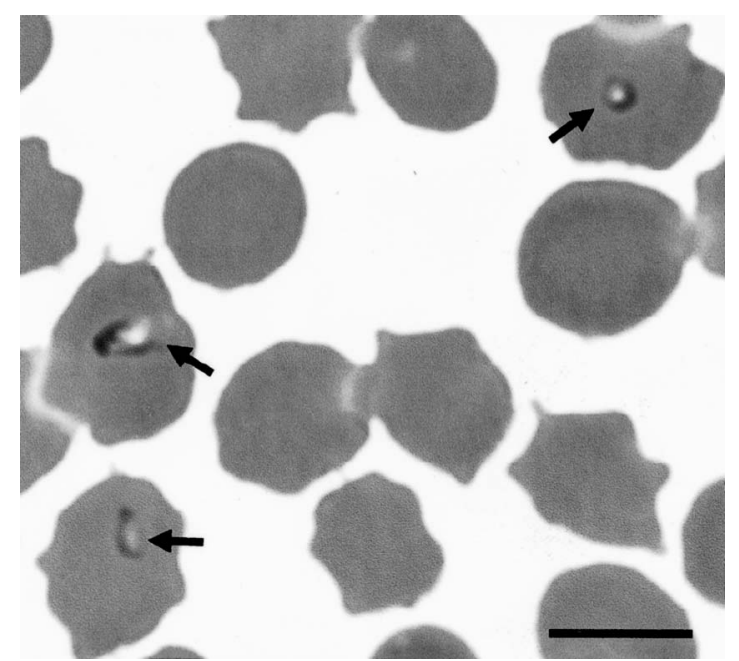

Figure 1. Photomicrograph of a stained blood film from cougar 1 with erythrocytes infected with Cytauxzoon felis organisms (arrows). Wright-Giemsa stain. Bar $=5$ $\mu \mathrm{m}$. 


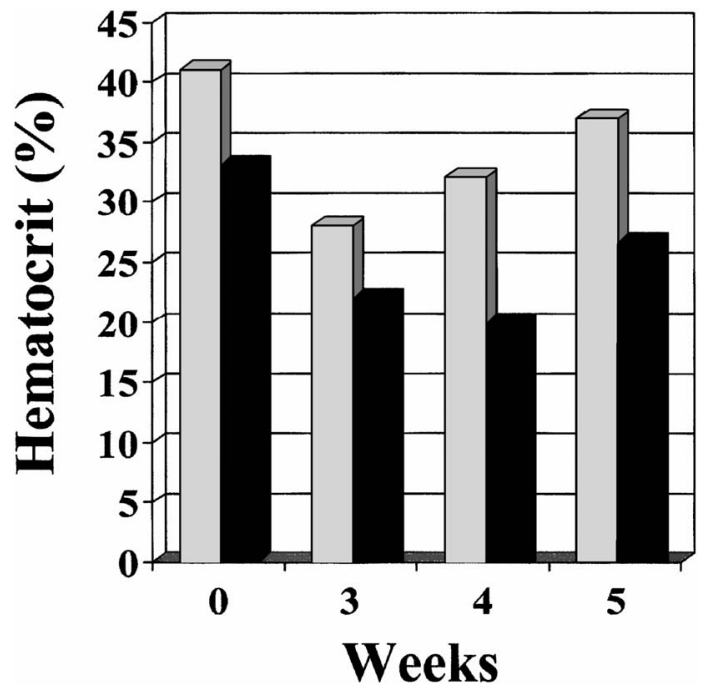

Figure 2. Hematocrits in cougar 2 (grey bars) and cougar 3 (black bars). Weeks represent the time after the animal began quarantine in Florida. Cytauxzoon organisms were not detected in stained blood films on the first sample date but were observed in stained blood films on the remaining dates.

tocol, animals were bled for laboratory analyses (CBC, Coulter Counter S+4, Coulter Electronic, Inc.; clinical chemistry panel, 550 Express Spectrophotometer, and 664 Fast 4 System, Ciba-Corning Diagnostics) at the University of Florida College of Veterinary Medicine after being anesthetized with a combination of ketamine hydrochloride and tiletamine-zolazepam. Piroplasms were not observed in erythrocytes on stained blood films when the animals were examined on arrival by a veterinary clinical pathologist (JWH), but organisms morphologically consistent with $C$. felis organisms were present in erythrocytes when examined 3,4 , and 5 wk later by the same veterinary clinical pathologist. An anemia developed concomitant with the appearance of piroplasms in erythrocytes (Fig. 2). Polychromasia was present in stained blood films at weeks 3 and 4, indicating the presence of reticulocytes. The mean cell volume (MCV) and red cell distribution width (RDW) also increased in response to the anemia (Figs. 3, 4). Leukocyte counts did not change that could be attributed to the parasitemia. Platelet counts were not done. However, examination of the stained blood films suggested that platelet numbers might have decreased slightly week 3. Serum bilirubin, (Fig. 5), ALT (Fig. 6), and AST (not shown) appeared to increase transiently week 3. In contrast, total protein (not shown) and globulin (Fig. 7) values where higher in serum from

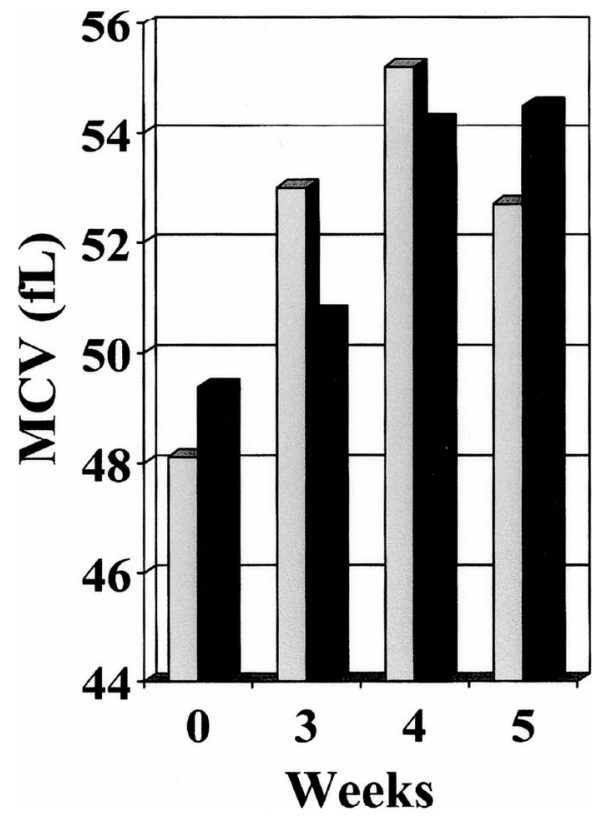

Figure 3. Mean cell volumes (MCV) in cougar 2 (grey bars) and cougar 3 (black bars). Weeks represent the time after the animal began quarantine in Florida. Cytauxzoon organisms were not detected in stained blood films on the first sample date but were observed in stained blood films on the remaining dates.

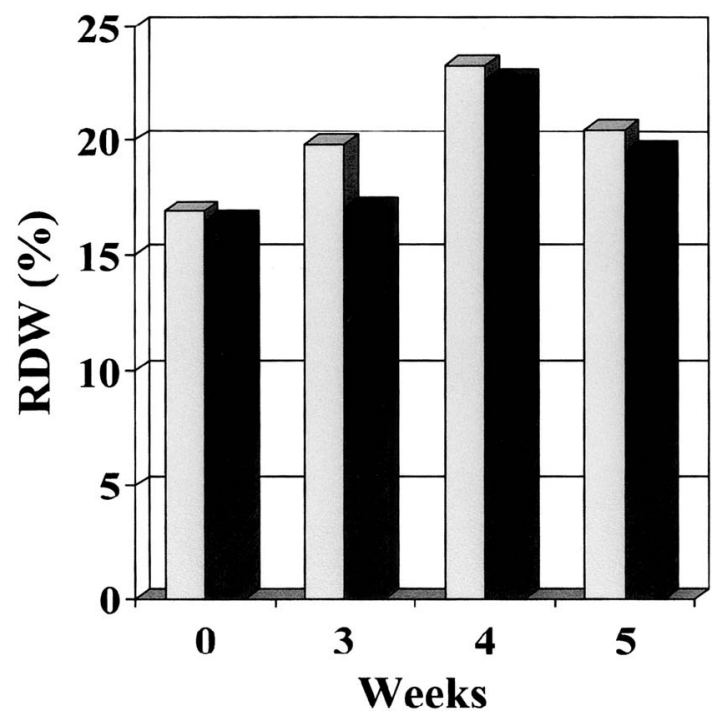

Figure 4. Red cell distribution width (RDW) in cougar 2 (grey bars) and cougar 3 (black bars). Weeks represent the time after the animal began quarantine in Florida. Cytauxzoon organisms were not detected in stained blood films on the first sample date but were observed in stained blood films on the remaining dates. 


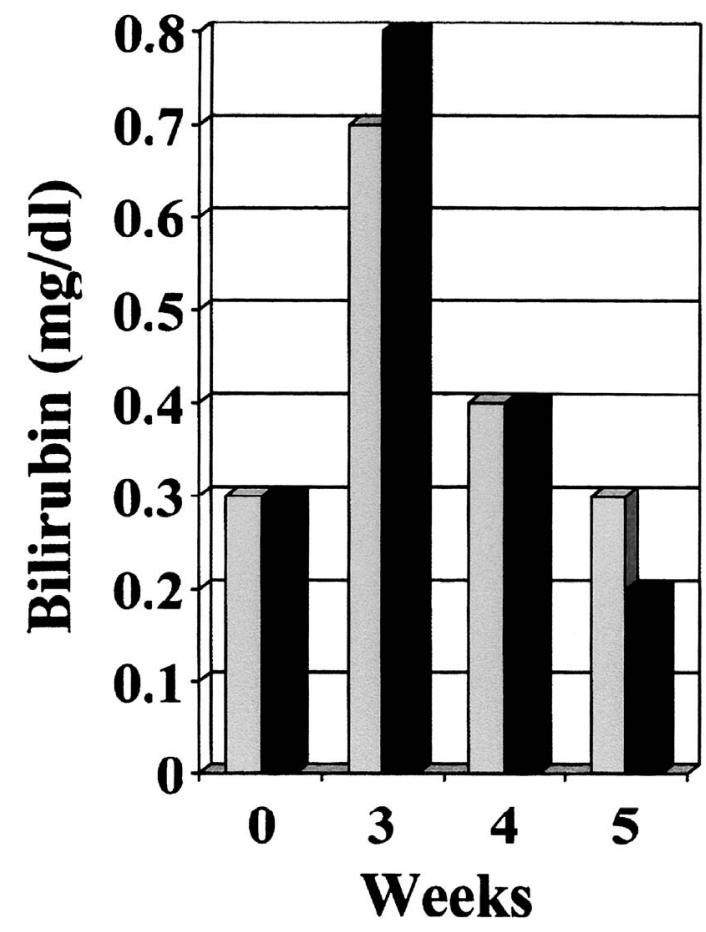

Figure 5. Serum bilirubin concentrations in cougar 2 (grey bars) and cougar 3 (black bars). Weeks represent the time after the animal began quarantine in Florida. Cytauxzoon organisms were not detected in stained blood films on the first sample date but were observed in stained blood films on the remaining dates.

weeks 3, 4, and 5 compared with serum collected on arrival. No other changes were apparent in clinical chemistry values that could be attributable to the infection. Animals were not noticeably ill during this quarantine period. Both cougars were subsequently radio-instrumented and released in southern Florida as part of the Florida cougar genetic restoration project. ${ }^{15}$ The presumptive infection of these western cougars with $C$. felis was not considered a risk to the free-ranging cougar population southern Florida because piroplasms (believed at that time to be $C$. felis) were already present in blood of at least one third of the free-ranging cougars without demonstrable evidence of disease attributable to this infectious agent. ${ }^{18}$ Cougar 2 died in August 1999 because of metabolic complications associated with pregnancy. Cougar 3 died in January 2001, and pneumonia was diagnosed at necropsy. ${ }^{15}$ EDTA-blood samples collected from cougars 2 and 3 during health screening and radio telemetry collaring in 1995 were frozen at $-20^{\circ} \mathrm{C}$ until molecular analysis was performed in 2005. Both blood samples were strongly positive for C. felis (and

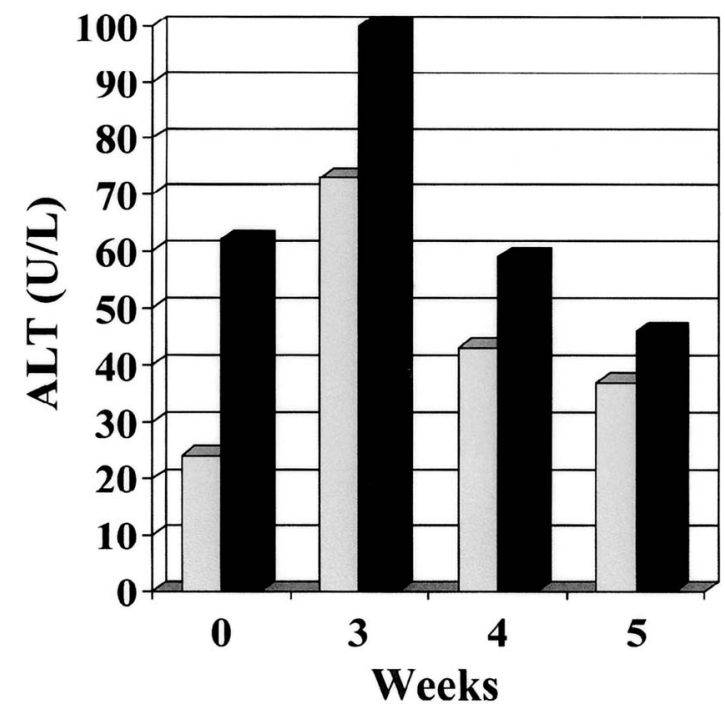

Figure 6. Serum alanine aminotransferase (ALT) activity in cougar 2 (grey bars) and cougar 3 (black bars). Weeks represent the time after the animal began quarantine in Florida. Cytauxzoon organisms were not detected in stained blood films on the first sample date but were observed in stained blood films on the remaining dates.

negative for the Babesia sp.) on the basis of PCR and sequencing of a fragment of the $18 \mathrm{~S}$ rRNA gene. ${ }^{23}$

\section{DISCUSSION}

The first clinical signs observed in domestic cats infected with $C$. felis are depression and anorexia. The rectal temperature rises gradually, at times to $41^{\circ} \mathrm{C}$ or more, during the 3 to 4 days after illness first becomes clinically apparent. Icterus, dehydration, and sometimes pale mucous membranes develop rapidly during the febrile period. Thereafter, the body temperature begins to drop, becoming subnormal in 2 to 3 days. Splenomegaly can be palpable. Dyspnea generally develops late in the course of the disease. Capillary refill time increases $(>2 \mathrm{sec})$. Experimental animals inoculated with infected tissue rarely live more than $3 \mathrm{wk}$ after inoculation, and cats are usually dead within 1 wk or less after clinical signs of illness are observed. ${ }^{10,11}$

In the terminal stages of the disease, most domestic cats will have icteric plasma. The hematocrit can be in the low 30s but is usually in the 20s or upper teens. Reticulocyte counts are not increased in response to the anemia. Cats become thrombocytopenic during late stages of disease. Leukocyte counts are variable, but leukopenia generally develops terminally. Coagulation tests (prothrombin time and activated partial prothrombin time) can be 


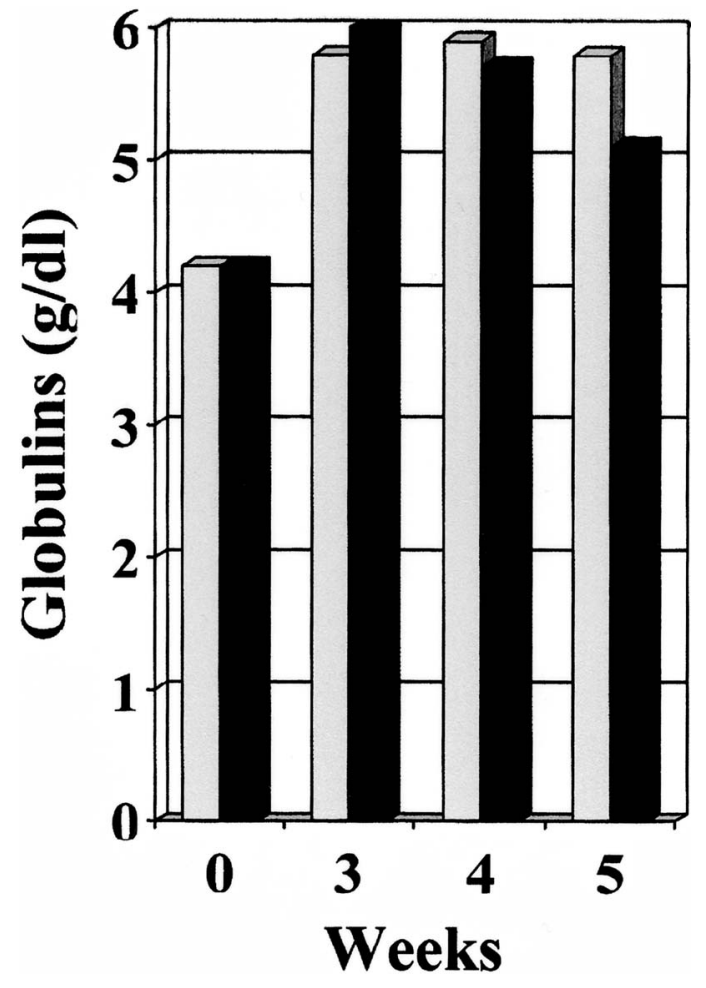

Figure 7. Serum globulin concentrations in cougar 2 (grey bars) and cougar 3 (black bars). Weeks represent the time after the animal began quarantine in Florida. Cytauxzoon organisms were not detected in stained blood films on the first sample date but were observed in stained blood films on the remaining dates.

prolonged or remain normal. Serum ALT, urea nitrogen, and glucose are frequently increased, and serum protein concentration is consistently decreased. Parasitemia occurs late in the disease. Organisms appear in erythrocytes as small round, oval, or bipolar bodies in blood films stained with Wright-Giemsa. The nucleus of the organism appears red to purple, and the cytoplasm stains a light blue. In the late stages of the disease, up to $25 \%$ of erythrocytes can contain organisms, but organisms can also be absent. $6,10,11$

Cougar 1 was ill with anorexia, depression, and dehydration, but he did not exhibit a high body temperature, icterus, or dyspnea. Splenomegaly was present when radiographed, but this was attributed to anesthesia. Clinical signs of illness were not recognized in cougars 2 and 3. Like these cougars, bobcats generally develop a nonfatal form of the disease..$^{9,14}$ Although evidence of illness was not recognized in several experimentally infected bobcats, one infected bobcat developed clinical signs and died. Necropsy findings from this bobcat were typical of fatal cytauxzoonosis in domestic cats. ${ }^{8,13}$ Fatal cytauxzoonosis has also been documented in a naturally infected 2-3-mo-old bobcat. ${ }^{17}$

The hematocrit decreased in all three cougars in this report concomitant with the first recognized parasitemia. The hematocrit would likely have been lower in cougar 1 because of the infection had the animal not been dehydrated at the time of examination. The initial decrease in hematocrit was associated with an increase in serum bilirubin in each case, suggesting that the anemia was the result of increased erythrocyte destruction, as occurs in cytauxzoonosis in domestic cats. There was no polychromasia in blood when parasitemia was first recognized, indicating a lack of reticulocyte response at that time. The presence of polychromatophilic erythrocytes in blood during the following $2 \mathrm{wk}$ in cougars 2 and 3 indicated the presence of a reticulocyte response to the anemia. A follow-up CBC was not done for cougar 1 until several months after a regenerative response would presumably have occurred. Assuming that an adequate supply of iron is present, reticulocytes and erythrocytes formed in response to anemia are larger than normal. The subsequent increases in MCV and RDW (an electronic measure of anisocytosis) values in cougars 2 and 3 are consistent with the production of a population of larger than normal cells in response to anemia. Although the anemia is typically nonregenerative in domestic cats with cytauxzoonosis, a regenerative reticulocytosis occurs in the rare animals that survive the initial infection. ${ }^{22}$ The low total leukocyte count and thrombocytopenia seen in cougar 1 are consistent findings in domestic cats with terminal cytauxzoonosis. These abnormalities were not present in cougars 2 or 3 .

The most consistent clinical chemistry findings in the cougars in this report were increased serum bilirubin, ALT, and AST at the time of initial parasitemia. The increased serum ALT indicates injury to hepatocytes, which could be the result of hypoxia from anemia, mechanical obstruction of blood flow by schizonts in pulmonary and hepatic vessels, or both. Serum AST is not a tissue-specific enzyme, but it can be increased with hepatocellular injury. The increased serum bilirubin could result from erythrocyte destruction, liver disease, or both. Serum protein findings were not consistent in these cougars. The low serum albumin present in cougar 1 is consistent with that reported with cytauxzoonosis in domestic cats. ${ }^{10}$ In contrast, serum albumin concentrations were relatively constant in cougars 2 and 3, but their serum globulin concentrations increased during quarantine. It is not known whether the increased globulin concentration was a result 
of C. felis infection or a response to one or more other antigens after arrival in Florida.

Although reinfection cannot be ruled out, these cougars appeared to have remained chronically infected with $C$. felis, as evidenced by positive molecular assays determined years (16 yr in cougar 1) after initial diagnosis of cytauxzoonosis was made by finding piroplasms in stained blood films. On the basis of current evidence, it appears that all or nearly all bobcats, cougars, and domestic cats that recover from acute $C$. felis infection remain parasitemic (probably for life). ${ }^{13,16}$

\section{CONCLUSIONS}

Although $C$. felis infection might not result in a decreased hematocrit in chronically infected cougars, in this report, we demonstrate that hemolytic anemia, and probably liver injury, occurs concomitant with the initial detection of $C$. felis organisms in stained blood films.

Acknowledgments: We thank Drs. Scott Citino and David Murphy for information about cougar 1, Ms. Anne Quinting for arranging for a blood sample from cougar 1, and Dr. Mark Cunningham for blood samples from cougars 2 and 3 .

\section{LITERATURE CITED}

1. Blouin, E. F., A. A. Kocan, B. L. Glenn, K. M. Kocan, and J. A. Hair. 1984. Transmission of Cytauxzoon felis Kier, 1979 from bobcats, Felis rufus (Schreber), to domestic cats by Dermacenter variabilis (Say). J. Wildl. Dis. 20: 241-242.

2. Blouin, E. F., A. A. Kocan, K. M. Kocan, and J. Hair. 1987. Evidence of a limited schizogonous cycle for Cytauxzoon felis in bobcats following exposure to infected ticks. J. Wildl. Dis. 23: 499-501.

3. Buergelt, C. D., B. L. Homer, and M. G. Spalding. 2002. Causes of mortality in the Florida panther (Felis concolor coryi). Ann. N. Y. Acad. Sci. 969: 350-353.

4. Butt, M. T., D. Bowman, M. C. Barr, and M. E. Roelke. 1991. Iatrogenic transmission of Cytauxzoon felis from a Florida panther (Felis concolor coryi) to a domestic cat (Felis domesticus). J. Wildl. Dis. 27: 342-347.

5. Culver, M., W. E. Johnson, J. Pecon-Slattery, and S. J. O'Brien. 2000. Genomic ancestry of the American puma (Puma concolor). J. Hered. 91: 186-197.

6. Franks, P. T., J. W. Harvey, R. P. Shields, and M.J.P. Lawman. 1988. Hematological findings in experimental feline cytauxzoonosis. J. Am. Anim. Hosp. Assoc. 24: 395-401.

7. Garner, M. M., N. P. Lung, S. Citino, E. C. Greiner, J. W. Harvey, and B. L. Homer. 1996. Fatal cytauxzoonosis in a captive-reared white tiger (Panthera tigris). Vet. Pathol. 33: 82-86.

8. Glenn, B. L., A. A. Kocan, and E. F. Blouin. 1983.
Cytauxzoonosis in bobcats. J. Am. Vet. Med. Assoc. 183: 1155-1158.

9. Glenn, B. L., R. E. Rolley, and A. A. Kocan. 1982. Cytauxzoon-like piroplasms in erythrocytes of wildtrapped bobcats in Oklahoma. J. Am. Vet. Med. Assoc. 181: 1251-1253.

10. Green, C. E., J. Meinkoth, and A. A. Kocan. 2006. Cytauxzoonosis. In: Greene, C. E. (ed.). Infectious Diseases of the Dog and Cat, 3rd ed. Saunders Elsevier, St. Louis, Missouri. Pp. 716-722.

11. Hoover, J. P., D. B. Walker, and J. D. Hedges. 1994. Cytauxzoonosis in cats: eight cases (1985-1992). J. Am. Vet. Med. Assoc. 205: 455-460.

12. Kier, A. B., J. E. Wagner, and L. G. Morehouse. 1982. Experimental transmission of Cytauxzoon felis from bobcats (Lynx rufus) to domestic cats (Felis domesticus). Am. J. Vet. Res. 43: 97-101.

13. Kier, A. B., S. R. Wightman, and J. E. Wagner. 1982. Interspecies transmission of Cytauxzoon felis. Am. J. Vet. Res. 43: 102-105.

14. Kocan, A. A., and E. F. Blouin. 1985. Hematologic and serum chemical value for free-ranging bobcats, Felis rufus (Schreber), with reference to animals with natural infections of Cytauxzoon felis Kier, 1979. J. Wildl. Dis. 21: $190-192$.

15. Land, D., D. Shindle, M. Cunningham, M. Lotz, and B. Ferree. 2004. Florida Panther Genetic Restoration and Management Annual Report. Florida Fish and Wildlife Conservation Commission, Naples, Florida.

16. Meinkoth, J., A. A. Kocan, L. Whitworth, G. Murphy, J. C. Fox, and J. P. Woods. 2000. Cats surviving natural infection with Cytauxzoon felis: 18 cases (19971998). J. Vet. Intern. Med. 14: 521-525.

17. Nietfeld, J. C., and C. Pollock. 2002. Fatal cytauxzoonosis in a free-ranging bobcat (Lynx rufus). J. Wildl. Dis. 38: 607-610.

18. Rotstein, D. S., S. K. Taylor, J. W. Harvey, and J. Bean. 1999. Hematologic effects of cytauxzoonosis in Florida panthers and Texas cougars in Florida. J. Wildl. Dis. 35: 613-617.

19. Taylor, S. K., C. D. Buergelt, M. E. Roelke-Parker, B. L. Homer, and D. S. Rotstein. 2002. Causes of mortality of free-ranging Florida panthers. J. Wildl. Dis. 38: 107-114.

20. Wagner, J. E. 1976. A fatal cytauxzoonosis-like disease in cats. J. Am. Vet. Med. Assoc. 168: 585-588.

21. Wagner, J. E., D. H. Ferris, A. B. Kier, S. R. Wightman, E. Maring, L. G. Morehouse, and R. D. Hansen. 1980. Experimentally induced cytauxzoonosis-like disease in domestic cats. Vet. Parasitol. 6: 305-311.

22. Walker, D. B., and R. L. Cowell. 1995. Survival of a domestic cat with naturally acquired cytauxzoonosis. J. Am. Vet. Med. Assoc. 206: 1363-1365.

23. Yabsley, M. J., T. L. Murphy, and M. W. Cunningham. 2006. Molecular detection and characterization of Cytauxzoon felis and a Babesia species in cougars from Florida. J. Wildl. Dis. 42: 366-374.

Received for publication 11 March 2005 